\title{
Generación de efluentes en el proceso de extracción de aceite crudo de Palma en el Ecuador
}

\author{
Generation of effluents in the process of extraction of \\ crude palm oil in Ecuador
}

Geração de efluentes no processo de extração de petróleo bruto de Palma no Equado

\author{
Paul N. Malacatus-Cobos ${ }^{\mathrm{I}}$ \\ pnmalacatus@uce.edu.ec \\ Byron V. Guerrero-Rodríguez II \\ bvguerreror@uce.edu.ec \\ Gerardo M. Llerena-Carrera ${ }^{\text {III }}$ \\ gmllerenac@uce.edu.ec
}

Recibido: 30 de enero de 2017 * Corregido: 20 de febrero de 2017 * Aceptado: 20 junio de 2017

\footnotetext{
I. Magister en Sistemas de Gestión; Ingeniero en Gestión Ambiental; Docente Ingeniería Ambiental; Universidad Central del Ecuador, Quito, Ecuador.

II. Master of Science in Leadership; Diploma Superior en Docencia Universitaria; Ingeniero Electrónico en Control y Automatización; Docente Ingeniería en Minas; Universidad Central del Ecuador, Quito, Ecuador.

III. Magister en Administración de Empresas (MBA) Programa Integral de Habilidades Múltiples; Ingeniero en Geología; Docente Ingeniería en Minas; Universidad Central del Ecuador, Quito, Ecuador.
} 


\section{Resumen}

La generación de aguas residuales en la industria extractora de aceite crudo de palma en el Ecuador actualmente no se tiene cuantificada, estas aguas son descardadas en los cuerpos de agua cercanos a cada una de las extractoras que por lo general, son los mismos cuerpos de agua de los cuales se realiza captación del recurso para el proceso productivo. Debido a que las extractoras de ubican en sectores rurales la gran mayoría de estos cuerpos de agua son utilizados por pobladores de la zona en diversas actividades.

El presente estudio se realizó en cinco extractoras de aceite de palma ubicadas en las provincias de Pichincha, Santo Domingo y Esmeraldas, el propósito fue establecer un valor promedio de generación de efluentes con relación a la cantidad de fruta fresca procesada en cada extractora, mismos que son tratados en sistemas de lagunas. Para lo cual se realizó la medición de caudales por el método de aforo volumétrico y los datos se analizaron estadísticamente.

Según los datos obtenidos mediante mediciones en campo en tres extractoras y análisis de información proporcionada por las dos restantes, se observa que la generación de efluentes varía en función de la capacidad de procesamiento y características de cada planta extractora. El valor promedio de agua residual generada por tonelada de RFF es de 0,84 m3/tRFF.

Palabras clave: Efluente; extractora de aceite; generación. 


\section{Abstract}

The generation of wastewater in the crude oil palm extracting industry in Ecuador is not currently quantified, these waters are discharged in bodies of water near each of the extractors that are usually the same bodies of water of which capture of the resource for the productive process is made. Because extractors are located in rural areas, the vast majority of these bodies of water are used by residents of the area in various activities.

This study was conducted in five extraction of palm oil in the provinces of Pichincha, Santo Domingo and Esmeraldas, the purpose was to establish an average value of effluent generation relative to the amount of fresh fruit processed in each extractor, same as they are treated in lagoon systems. For which the flow measurement by the method of volumetric capacity and the data were analyzed statistically performed.

According to the data obtained through field measurements in three extraction and analysis of information provided by the other two, it is observed that effluent generation varies depending on processing capacity and characteristics of each extraction plant. The average value of wastewater generated per tonne of FFB is $0.84 \mathrm{~m} 3 / \mathrm{tRFF}$.

Key words: Effluent; oil extraction; generation. 


\section{Introducción.}

La generación de efluentes en el proceso de extracción de aceite de palma se relaciona con la capacidad de procesamiento de la planta. [1] En Ecuador no se ha determinado un valor referencial de generación de efluente por tonelada de racimo de fruta fresca procesada (tRFF), el cual en Colombia se estimó en 0,84m3/tRFF.

En el presente estudio se analizaron datos de cinco plantas extractoras de aceite de palma ubicadas en la zona noroeste del país con el objetivo de establecer un valor promedio de generación de agua residual en relación a las toneladas de fruta fresca procesada. Este valor servirá para optimizar el consumo de agua, estimar la generación de efluentes y considerar de manera preliminar las dimensiones de los sistemas de tratamiento. Adicionalmente sería un valor referente para estudios con mayor alcance y profundidad en este sector.

Es necesario mencionar que para este estudio se consideró solo la producción de aceite rojo de palma, excluyendo los procesos de palmistería que realizan algunas de estas empresas, debido a que los procesos son sustancialmente diferentes tanto en proceso como en el consumo de agua y generación de efluentes.

De manera general, el proceso productivo se describe en la figura 1. 


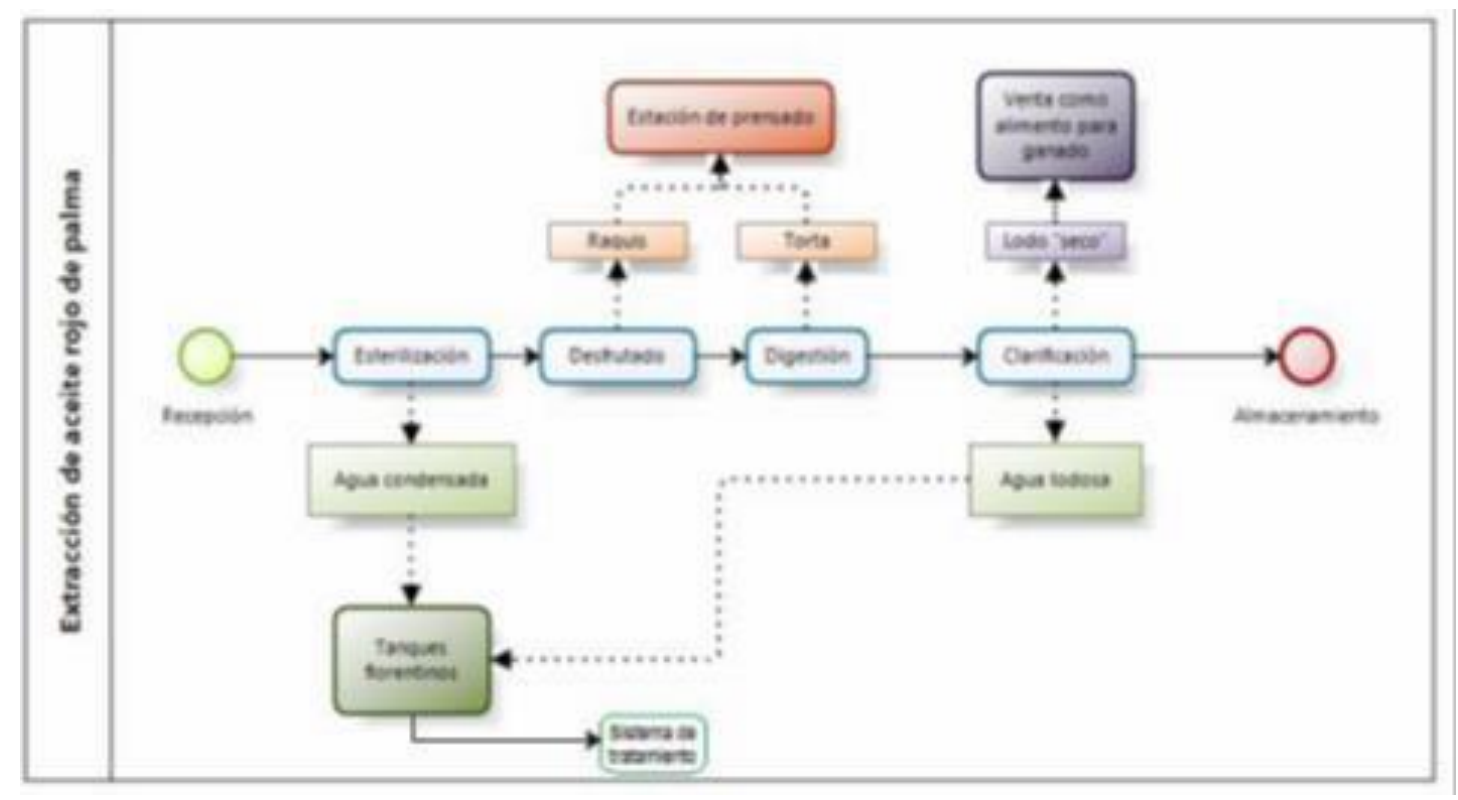

Figura 1. Diagrama de flujo del proceso y destino de efluentes. [1]

Recepción del fruto: consiste en pesar el camión lleno de fruta proveniente de los productores agrícolas y luego de descargarlo para obtener por diferencia el peso neto de la fruta, en este proceso generalmente se realiza un control de calidad de la fruta que permite también determinar en algunos casos porcentajes de perdida de materia prima.

Esterilización: se lleva a cabo sometiendo los racimos de fruto fresco de palma a la acción de vapor de agua en autoclaves, donde el tiempo de cocción y la temperatura se ajustan dependiendo del tamaño de los racimos y del grado de madurez de los mismos, esta es una de las fases del proceso que genera mayor cantidad de efluentes.

Desfrutado: se procede a separar el fruto del racimo en un tambor rotatorio, el fruto se separa para luego enviarlo al digestor y el racimo sin frutos se utiliza como abono orgánico. 
Digestión: El fruto es depositado en cilindros llamados digestores que presentan unas paletas en las cuales va a macerar el fruto por medio de la agitación circular, además se le aplica vapor, esto ayuda a que las células de aceite se desprendan del fruto y la recuperación del aceite en el momento del prensado sea eficiente.

Prensado: El fruto ya digestado se procede a prensarlo. En esta etapa se le aplica agua a la salida del digestor y en la parte inferior de la prensa con el fin de lavar las fibras y lograr que la extracción del aceite sea lo más eficientemente posible y mantener las pérdidas de aceite dentro de los estándares, además de dar la dilución adecuada para realizar la separación en la sección de clarificación, la fibra alimenta al caldero puesto se utiliza la misma como combustible, de igual forma se realiza la recuperación de la nuez que pasara al proceso de palmisteria en algunos caso o en otros al almacenamiento y posterior transporte así otras plantas para su proceso.

Clarificación: mediante la clarificación se remueven sólidos insolubles y disueltos en el agua que contiene el aceite. Está conformado por un tanque clarificador continuo de aceite, diseñado para separar el aceite del agua y los sólidos con los cuales están mezclados, al salir del tanque de aceite crudo después del tamiz vibratorio obrando en una forma continua. Se utiliza también centrifugas de tres fases que separan como etapa final el aceite, lodos y agua, la cual va al sistema de tratamiento, en el caso de los lodos se los utiliza como sobre alimento del ganado.

Almacenamiento: Una vez que el aceite alcanza los niveles de pureza requerido es enviado a los tanques de almacenamiento para su posterior despacho, de igual forma se realizan controles de calidad al aceite que se despacha hacia los proveedores. 
Todos los procesos donde se utiliza agua y que realizan aporte de efluentes al sistema de tratamiento de aguas residuales se muestran en la tabla 1.

Tabla 1: Efluentes originados en el proceso para la extracción de aceite rojo y su destino. [1]

\begin{tabular}{cll}
\hline Etapa del proceso & \multicolumn{1}{c}{ Efluente } & $\begin{array}{c}\text { Destino del } \\
\text { efluente }\end{array}$ \\
\hline Recepción & $\begin{array}{l}\text { Impureza de la } \\
\text { fruta }\end{array}$ & $\begin{array}{l}\text { Plantaciones, } \\
\text { como abono }\end{array}$ \\
Esterilización & Líquidos & $\begin{array}{l}\text { Tratamiento de } \\
\text { aguas (lagunas) }\end{array}$ \\
& Raquis vacíos & Plantaciones, \\
como abono
\end{tabular}

\section{Elaborado por: Autores}

\section{Fuente: Datos obtenidos en campo}

El agua residual es transportada al sistema de tratamiento que en la mayoría de los casos cuenta con una etapa previa de recuperación de aceite en tanques florentinos, los cuales consisten en unas trampas de grasa y sedimentadores. 


\section{Materiales y métodos.}

Para el levantamiento de información para medir las variables del estudio se plantearon como: la generación de efluentes industriales en m3 y la tonelada de fruta fresca procesada tRFF, se realizaron visitas de campo a tres extractoras, durante las cuales se estudió el proceso productivo, se recopiló el historial de producción y se midió el caudal de ingreso al sistema de tratamiento de aguas residuales. Las dos extractoras restantes proporcionaron datos de su historial de producción y consumo de agua.

Para la medición de caudales se aplicó la metodología de Romero Rojas, que señala: para la medición de caudales se debe realizar por lo menos tres jornadas de medición y muestreo horario de 24 horas de duración [3]. La medición en cada extractora se ajustó a las políticas de seguridad de cada empresa, realizando así entre once y trece mediciones diarias por el método de aforo volumétrico.

Los datos obtenidos se analizaron estadísticamente de acuerdo a la frecuencia de ocurrencia [1] y graficando los resultados, se obtuvo una buena correlación a una recta sobre papel log normal de probabilidad, por lo tanto la distribución es log normal y la media corresponde mejor al promedio geométrico.

El valor promedio de caudal $(\mathrm{m} 3 / \mathrm{h})$ se relacionó con la producción de la planta (tRFF/h), obteniendo un valor de generación de efluente en m3/tRFF. 


\section{Resultados y discusión.}

A continuación se presentan los resultados obtenidos en las 5 extractoras de palma objeto del estudio:

Tabla 2: Generación de efluentes

\begin{tabular}{|c|c|c|c|}
\hline Extractora & $\begin{array}{c}\text { Caudal } \\
(\mathbf{m 3 / h})\end{array}$ & $\begin{array}{c}\text { Producción } \\
(\mathbf{t R F F} / \mathbf{h})\end{array}$ & $\begin{array}{c}\text { Generación } \\
\text { efluente } \\
(\mathbf{m 3} / \mathbf{t R F F})\end{array}$ \\
\hline $\mathbf{E 1}$ & 9,98 & 17,48 & 0,57 \\
\hline $\mathbf{E 2}$ & 32,98 & 39,41 & 0,84 \\
\hline $\mathbf{E 3}$ & 11,42 & 19,34 & 0,59 \\
\hline$* \mathbf{E} 4$ & 6,80 & 9.1 & 0,94 \\
\hline$* \mathbf{E 5}$ & 6,90 & 8,97 & 1,28 \\
\hline \multicolumn{3}{|c|}{ Promedio } & $\mathbf{0 , 8 4}$ \\
\hline
\end{tabular}

*Valores proporcionados por la empresa.

Elaborado por: Autores

\section{Fuente: Datos obtenidos en campo y empresas}

El resultado obtenido de generación de efluentes en promedio de las 5 extractoras es de 0,84 $\mathrm{m} 3 / \mathrm{tRFF}$.

Este resultado corrobora el promedio obtenido por el estudio de CENIPALMA Colombia, que es $0,84 \mathrm{~m} 3 / \mathrm{tRFF}$.

Si consideramos que en el Ecuador se produjeron aproximadamente 2’823.180 TM de fruta de palma en el año 2016, de acuerdo a lo establecido por FEDAPAL 2016, podríamos decir, de 
acuerdo al dato obtenido en este estudio de generación de efluentes industriales que se generaron aproximadamente 2`371.471,2 m3 de efluentes de la industria en el año 2016.

De acuerdo a los datos se tiene que la generación de efluentes por tonelada de fruta procesada se relaciona también con el control de operaciones en el proceso productivo ya que las empresas que aplican producción más limpia generan menos efluentes.

Además de la extracción de aceite algunas de las empresas realizan también procesos de palmistería que a su vez generan más efluente que se incorpora al mismo sistema de tratamiento, siendo necesario analizar cada caso particular antes de dimensionar sistemas de tratamiento.

\section{Conclusiones.}

El valor promedio de generación de efluentes por tonelada de racimo de fruta fresca procesada en las cinco extractoras analizadas es $0,84 \mathrm{~m} 3$.

Las etapas del proceso que generan mayor cantidad de efluente son: esterilización y extracción. Siendo estos los puntos críticos para el control y optimización del uso de agua.

En el Ecuador se produjeron aproximadamente 2`371.471,2 m3 de efluentes de la industria de extracción de aceite crudo de palma en el año 2016, de acuerdo a las estadísticas de producción y el dato de generación de efluentes obtenido en el presente estudio.

\section{Recomendaciones.}

Para obtener un valor referencial a nivel nacional con un porcentaje menor de error, se debe ampliar el alcance del estudio tanto en número de extractoras como haciendo una 
distinción en los procesos productivos de las mismas, de forma que se pueda manejar estadísticas de generación de efluentes de acuerdo al tipo de proceso productivo de la extractora y el alcance de extracción tanto de aceite crudo de palma y de aceite de palmiste.

Implementar sistemas de cuantificación de generación de efluentes industriales tanto mecánicos y/o automáticos en las extractoras de aceite crudo de palma de forma que se obtenga datos más específicos de la temática.

\section{Bibliografía.}

[1]. J. A. García Montes, «Estado actual del manejo de efluentes en Colombia,» Palmas, vol. 14, $\mathrm{n}^{\mathrm{o}}$ Especial, pp. 141-158, 1993.

[2]. E. V. Chamorro Cuásquer y G. M. Orellana Lara, Análisis de eficiencia del sistema de tratamiento de aguas residuales de la extractora de aceite de palma La Sexta S.A. y propuesta de rediseño de las lagunas facultativas, Quito, 2016.

[3]. J. A. Romero Rojas, Tratamiento de Aguas Residuales. Teoría y principios de diseño., Tercera ed., Bogotá: Escuela Colombiana de Ingeniería, 1999.

[4]. M. Bonomie y M. Reyes, «Estrategia ambiental en el manejo de efluentes en la extracción de aceite de palma,» TELOS, Revista Interdisciplinaria en Ciencias Sociales, vol. 14, pp. 323 - 332, 2012. 